\title{
Learning to predict pain: differences in people with persistent neck pain and pain-free controls
}

\author{
Daniel S Harvie ${ }^{\text {Corresp., 1, } 2}$, Jeroen D Weermeijer ${ }^{3}$, Nick A Olthof ${ }^{1,2}$, Ann Meulders $^{3,4,5}$ \\ ${ }^{1}$ The Hopkins Centre, Menzies Health Institute Queensland, Griffith University, Gold Coast, QLD, Australia \\ 2 School of Allied Health Sciences, Griffith University, Gold Coast, QLD, Australia \\ ${ }^{3}$ Center for Contextual Psychiatry, Katholieke Universiteit Leuven, Leuven, Belgium \\ 4 Research Group Health Psychology, Katholieke Universiteit Leuven, Leuven, Belgium \\ 5 Experimental Health Psychology, Maastricht University, Maastricht, Limburg, Netherlands \\ Corresponding Author: Daniel S Harvie \\ Email address: d.harvie@griffith.edu.au
}

Background. Learning to predict threatening events enables an organism to engage in protective behavior and prevent harm. Failure to differentiate between cues that truly predict danger and those that do not, however, may lead to indiscriminate fear and avoidance behaviors, which in turn may contribute to disability in people with persistent pain. We aimed to test whether people with persistent neck pain exhibit contingency learning deficits in predicting pain relative to pain-free, gender- and age-matched controls.

Method. We developed a differential predictive learning task with a neck pain-relevant scenario. During the acquisition phase, images displaying two distinct neck positions were presented, and participants were asked to predict whether these neck positions would lead to pain in a fictive patient with persistent neck pain (see fictive patient scenario details in Appendix A). After participants gave their painexpectancy judgment in the hypothetical scenario, the verbal outcome (PAIN or NO PAIN) was shown on the screen. One image (CS+) was followed by the outcome 'PAIN', while another image (CS-) was followed by the outcome 'NO PAIN'. During the generalization phase, novel but related images depicting neck positions along a continuum between the CS+ and CS- images (generalization stimuli; GSs) were introduced to assess the generalization of acquired predictive learning to the novel images; the GSs were always followed by the verbal outcome "NOTES UNREADABLE" to prevent extinction learning. Finally, an extinction phase was included in which all images were followed by 'NO PAIN' assessing the persistence of pain-expectancy judgments following disconfirming information.

Results. Differential pain-expectancy learning was reduced in people with neck pain relative to controls, resulting from patients giving significantly lower pain-expectancy judgments for the CS+, and significantly higher pain-expectancy judgments for the CS-. People with neck pain also demonstrated flatter generalization gradients relative to controls. No differences in extinction were noted.

Discussion. The results support the hypothesis that people with persistent neck pain exhibit reduced differential pain-expectancy learning and flatter generalization gradients, reflecting deficits in predictive learning. Contrary to our hypothesis, no differences in extinction were found. These findings may be relevant to understanding behavioral aspects of chronic pain. 
1

2 Learning to predict pain: differences in people with 3 persistent neck pain and pain-free controls

4

5 Daniel S. Harvie ${ }^{1,2,3}$, Jeroen D. Weermeijer ${ }^{3}$, Nick A. Olthof ${ }^{1,2}, \&$ Ann Meulders ${ }^{5,6}$

$7 \quad{ }^{1}$ The Hopkins Centre, Menzies Health Institute Queensland, Griffith University, Gold Coast, Australia

$8{ }^{2}$ School of Allied Health Sciences, Griffith University, Gold Coast, Australia

$9 \quad{ }^{3}$ Center for Contextual Psychiatry, Katholieke Universiteit Leuven, Leuven, Belgium

$10{ }^{4}$ Research Group Health Psychology, Katholieke Universiteit Leuven, Leuven, Belgium

$11{ }^{5}$ Experimental Health Psychology, Maastricht University, Maastricht, The Netherlands

12

Corresponding Author:

Dr. Daniel Harvie, The Hopkins Centre, Menzies Health Institute QLD, Griffith University Gold Coast

Campus, Parklands Drive, Southport Australia QLD 4222. 


\section{Abstract}

Background. Learning to predict threatening events enables an organism to engage in protective behavior and prevent harm. Failure to differentiate between cues that truly predict danger and those that do not, however, may lead to indiscriminate fear and avoidance behaviors, which in turn may contribute to disability in people with persistent pain. We aimed to test whether people with persistent neck pain exhibit contingency learning deficits in predicting pain relative to painfree, gender- and age-matched controls.

Method. We developed a differential predictive learning task with a neck pain-relevant scenario. During the acquisition phase, images displaying two distinct neck positions were presented, and participants were asked to predict whether these neck positions would lead to pain in a fictive patient with persistent neck pain (see fictive patient scenario details in Appendix A). After participants gave their pain-expectancy judgment in the hypothetical scenario, the verbal outcome (PAIN or NO PAIN) was shown on the screen. One image (CS+) was followed by the outcome 'PAIN', while another image (CS-) was followed by the outcome 'NO PAIN'. During the generalization phase, novel but related images depicting neck positions along a continuum between the CS+ and CS- images (generalization stimuli; GSs) were introduced to assess the generalization of acquired predictive learning to the novel images; the GSs were always followed by the verbal outcome "NOTES UNREADABLE" to prevent extinction learning. Finally, an extinction phase was included in which all images were followed by 'NO PAIN' assessing the persistence of pain-expectancy judgments following disconfirming information.

Results. Differential pain-expectancy learning was reduced in people with neck pain relative to controls, resulting from patients giving significantly lower pain-expectancy judgments for the CS+, and significantly higher pain-expectancy judgments for the CS-. People with neck pain also 
42 demonstrated flatter generalization gradients relative to controls. No differences in extinction were

43 noted.

44 Discussion. The results support the hypothesis that people with persistent neck pain exhibit

45 reduced differential pain-expectancy learning and flatter generalization gradients, reflecting

46 deficits in predictive learning. Contrary to our hypothesis, no differences in extinction were found.

47 These findings may be relevant to understanding behavioral aspects of chronic pain.

48 Key words: Generalization, extinction, neck pain, chronic pain, predictive learning, contingency

49 learning 


\section{Introduction}

52 Learning to predict threatening events is highly adaptive as it enables an organism to

53 respond in anticipation with protective action to avoid or limit injury (Enquist, Lind, \& Ghirlanda,

54 2016). A prime mechanism underlying the prediction of threatening events is associative learning,

55 and more specifically classical conditioning (Pavlov, 1927). The outcome of an aversive

56 conditioning or fear conditioning procedure is apparent when an initially inert stimulus

57 (conditioned stimulus; CS) such as a neutral movement, comes to elicit defensive behavioral

responses (conditioned response; CR) such as increased autonomic arousal, verbal fear reports,

and avoidance behavior, after contingent pairing with an aversive stimulus such as pain (unconditioned stimulus; US) that innately elicits a response (unconditioned response; UR).

Contemporary biopsychosocial models of (musculoskeletal) chronic pain consider painrelated fear and avoidance as key contributors in the transition from acute to chronic pain (Meulders, 2019; Vlaeyen, 2015). Previous research indeed revealed that high scores on painrelated fear and avoidance beliefs questionnaires are associated with poor outcomes following injury and increased disability in a range of conditions such as neck pain (Landers, Creger, Baker, \& Stutelberg, 2008; Nederhand, Ijzerman, Hermens, Turk, \& Zilvold, 2004) and back pain (Crombez, Vlaeyen, Heuts, \& Lysens, 1999; for review see Vlaeyen \& Linton, 2012). These data suggest that it is not merely the severity of the injury and the associated pain intensity, but rather an individual's behavioral response to pain that paves the way to chronification. Given that injuryrelated learning shapes these individual responses to pain, learning processes may also be involved in determining functional outcomes. Following this reasoning, aberrant conditioning responses in people with persistent pain may exist when compared to people without persistent pain, which in turn may contribute to their vulnerability (Meulders, 2019). 

adaptive pain-related fear conditioning is learning to respond selectively to predictors of harm thus equally important is safety learning, which is, learning to refrain from responding to cues that do not predict harm. Failure to differentiate between danger and safety cues appears to be characteristic of pathology in generalized anxiety disorder (Duits et al., 2015; Grillon, 2002), post-traumatic stress disorder (Jovanovic, Kazama, Bachevalier, \& Davis, 2012; Lissek et al., 2005) schizophrenia (Clifton et al., 2017), and obsessive compulsive disorder (Apergis-Schoute et al., 2017) where affective and behavioral defensive responses are unduly triggered. and generalization towards novel situations and stimuli. Stimulus generalization (Honig \& Urcuioli, 1981; Kalish, 1969) allows extrapolating the acquired value of one stimulus to novel stimuli (generalization stimuli; GSs) based on (non-)perceptual similarities and has the advantage that defensive action can be taken in the absence of prior experience or new learning. Excessive fear generalization to safe situations however may induce persistent anticipatory anxiety and excessive avoidance behavior initiating a pathway towards disability (Dymond, Dunsmoor, Vervliet, Roche, \& Hermans, 2015; Meulders et al., 2014; Meulders, Meulders, Stouten, De Bie, \& Vlaeyen, 2017).

In recent years, associative learning deficits have been reported in several chronic pain populations, including fibromyalgia (Jenewein et al., 2013; Meulders, Jans, \& Vlaeyen, 2015; Meulders, Meulders, Stouten, De Bie, \& Vlaeyen, 2017), chronic unilateral hand pain (Meulders et al., 2014), and chronic back pain (Klinger et al., 2010; Schneider, Palomba, \& Flor, 2004; for review see Harvie, Moseley, Hillier, \& Meulders, 2017). In line with the anxiety literature, 
97 exaggerated conditioned responses to pain-associated cues $(\mathrm{CS}+)$ but rather impaired safety

98 learning (i.e. greater pain-expectancy and fear in response to safety cues (CS-)) as well as

99 "overgeneralization" (i.e. greater pain-expectancy and fear in response to novel stimuli that share

100 features with the pain-associated cues). Impaired predictive learning and stimulus generalization

101 may not only become maladaptive when it spreads excessively to safe stimuli, but also when it

102 persists despite disconfirmation of the expected outcome. Indeed previous studies suggests that

103 some patients may show resistance to extinction of differential conditioning (Schneider et al.,

104 2004) or generalization (Meulders et al., 2017) following corrective feedback, which may further

105 maintain disability in chronic pain conditions.

106 Second only to back pain, neck pain is the greatest cause of years lived with disability

107 worldwide (Vos et al., 2015). Yet, it is unknown whether associative learning deficits are a feature

108 of all or only specific persistent pain conditions, and thus it is unclear whether they are prevalent

109 among people with persistent neck pain. To date, it is commonly accepted that the conditions for

110 learning a causal relationship between two neutral events closely resemble those that underlie

111 classical conditioning with a biologically significant US (Dickinson, 1980; Shanks \& Dickinson,

112 1988). As such, contingency learning or predictive learning tasks, in which participants are

113 required to give outcome-expectancy judgments when presented with certain cues, offer a valid

114 proxy to tap into the basic processes underlying classical fear conditioning (Boddez et al., 2013).

115 Therefore, in line with previous studies using contingency learning tasks in chronic pain

116 populations (Meulders et al., 2014; Meulders et al., 2015; Meulders et al., 2017), we developed a

117 predictive learning task based around a clinical neck pain scenario to investigate whether people

118 with persistent neck pain show deficits in predictive learning relative to pain-free, age- and gender-

119 matched controls. We hypothesized that people with persistent neck pain would show (1) less 
120

121

122

123

124

125

126

\section{Materials and methods}

differential predictive learning at the end of acquisition (i.e. a reduced difference in CS+ vs. CS-

pain-expectancy judgments), (2) overgeneralization of pain-expectancy (e.g. flatter generalization gradients and higher pain-expectancy judgments for GSs more similar to the CS-) and (3) delayed extinction of acquired differential pain-expectancies (i.e. persistent differential CS+ vs. CS- painexpectancy) relative to healthy controls.

\section{Participants}

Thirty individuals with persistent neck pain $(14$ males, mean age $=50.9$ years, $S D$ age $=15.6)$ with diagnosis confirmed by a qualified physiotherapist, and 30 age- and gender-matched painfree controls (14 males, mean age $=49.9$ years, $S D$ age $=14.6)$ were recruited; the target sample size of 60 was a priori determined based on previous literature. That is, similar studies in other

133 pain groups have reported moderate effects $\left(\eta_{p}^{2}=0.07\right)$ for chronic pain vs. healthy control differences in pain-expectancy learning (Meulders, Jans, \& Vlaeyen, 2015). Using this effect size, we calculated that 30 subjects would be needed for $80 \%$ power to detect an interaction between stimulus type and group. To assist in accounting for multiple analyses we doubled the calculated sample size. Other studies have found significant between group effects for similar outcomes with just 48 subjects, providing further sample size support (Meulders et al., 2014). On average, included participants had moderate pain (mean pain intensity $=4.5, S D=1.2$ on a 11-point scale) and reported moderate disability (mean disability $=30 \%, S D=12 \%$ ) as assessed by the Neck 
142 disability (Vernon \& Mior, 1991). Participation was voluntary, and no monetary incentive was

143 provided. Participants with persistent neck pain were required to have had neck pain for a period

144 of at least 3 months, whilst control participants were required to have no neck or other pain, and

145 to have no history of pain lasting longer than 3 months. Participants in both groups were excluded

146 on the basis of neurological deficits (i.e., stroke, dementia, Alzheimer, cervical radiculopathy,

147 cervical myelopathy), diagnosed psychological disorders (i.e., PTSD, Schizophrenia, Bi-polar

148 Disorder, Dissociative Disorder, Panic Disorder), serious orthopaedic deficit that may indicate

149 ongoing physical driver of pain and disability (i.e. fracture, spondylolisthesis), or were currently

150 taking certain medications that might alter cognitive functioning (i.e. opioids, neuroleptics, and

151 anxiolytics). All participants were further required to have normal or corrected to normal vision.

152 Participants with neck pain were recruited through the Recover Injury Research Centre database

153 (Gold Coast, Australia), local clinics (Gold Coast, Australia), word-of-mouth, and social media.

154 Patients were asked to nominate a same-aged (+/- 5 years) friend or family member of the same

155 gender to volunteer as a control subject. Where participants were not able to bring an age- and

156 gender-matched control, participants were supplemented through recruitment posters placed

157 around the university campus, word-of-mouth, and social media. Initial contact and eligibility

158 screening was undertaken by telephone. Each participant provided written informed consent before

159 the start of the experiment. The protocol was approved by the Griffith University institutional

160 Human Research Ethics Committee (GFU Reference number: 2017/710). The protocol and

161 analysis plan were not preregistered. After the experiment, we assessed the following

162 psychological traits using questionnaires for descriptive purposes: (1) positive and negative affect

163 (Positive and Negative Affect Schedule; PANAS) (Watson, Clark, \& Tellegen, 1988), (2) trait 164 anxiety (State-Trait Anxiety Inventory; STAI) (Spielberger, Gorsuch, Lushene, Vagg, \& Jacobs, 
165 1983), (3) pain catastrophizing (Pain Catastrophizing Scale; PCS) (Sullivan, Bishop, \& Pivik, 166 1995), (4) fear of movement (Tampa Scale for Kinesiophobia; TSK-11) (Woby, Roach, Urmston, 167 \& Watson, 2005), and general health (Patient Health Questionnaire; PHQ-9) (Kroenke, Spitzer, \& 168 Williams, 2001). Participants also rated their current pain, habitual, and highest pain experienced 169 in the last two weeks. A detailed overview of the sample characteristics can be found in Table 1.

**INSERT UPDATED TABLE $1 * *$

171

172

\section{Software and stimulus material}

173 This study was programmed using Affect 4.0, a Windows-based freely available experimental software package (Spruyt, Clarysse, Vansteenwegen, Baeyens, \& Hermans, 2010). The cues that

175 served as conditioned stimuli (CS+ and CS-), and the generalization stimuli (GS1, GS2, GS3, GS4, and GS5) consisted of images of an avatar displaying different neck positions (Figure 1). The

177 images were created with the free 3-D animation software, DAZ studio 4.9 pro 178 (https://www.daz3d.com). The CS+ and CS- images were set at $75^{\circ}$ of head rotation in opposing directions. The GS1-5 images set at $25^{\circ}$ increments from $50^{\circ}$ left rotation to $50^{\circ}$ right rotation, starting in the direction of the CS+. All stimuli were presented on a 17-inch computer monitor, at the center of the screen on a black background. The outcome during acquisition was one of two verbal labels displayed on the computer screen, in font Arial with font size 48: "PAIN" and "NO

183 PAIN", during the generalization phase the following outcome was presented "NOTES 184 UNREADABLE', 
188 Figure 1. Conditioned stimuli (CS+ and CS-) and generalization stimuli (GS1-5) presented with

189 CSs on the extremes and the intermediate GSs in between representing the generalization gradient.

190 Which stimulus served as the CS+ and the CS- (i.e. white vs. blue labels) was counterbalanced

191 across participants.

192

193 Procedure

194 Data collection was undertaken in a laboratory environment, free from noise and visual distraction.

195 The task was undertaken while seated in front of a laptop with a 17 " screen, placed on a table 196 against a blank wall. The experimenter remained behind a curtain out of view. On arrival, written 197 information was provided, and participants signed informed consent. Upon starting the 198 computerized task, participants read a set of on-screen standardized instructions (Supplementary

199 File 1), during which they could ask for clarification. Once the instructions had been read and 200 questions answered, the computerized task was commenced. The experimenter (JDW or NO) 201 would remain inside the room but out of view. The entire experimental session took approximately 20230 minutes including questionnaires.

203

204

Neck pain scenario predictive learning task

205 Based on previous work (Meulders et al., 2014), we designed a predictive learning task based 206 around a clinical neck pain scenario (Figure 2). During the task, participants were asked to predict 207 whether certain neck positions would lead to pain in a fictive chronic neck pain patient, rather than 208 in themselves. At the start of each trial, an avatar displaying a neck position was presented. After $2092 \mathrm{~s}$, the question 'To what extent do you expect Alex to experience pain in this position?' appeared 210 above the image. Additionally, an 11-point numerical rating scale (NRS), ranging from 0 'not at 
211 all' to 10 'very much', was presented horizontally at the top of the screen, below the presented

212 question. Participants answered this question by clicking on a value with the left mouse button;

213 their choice was visualized by a white dot appearing on the selected value of the NRS. Participants

214 could then confirm their answer by pressing the spacebar. After answer confirmation, the image

215 would disappear, followed by one of the three possible outcomes: 'PAIN', 'NO PAIN', or 'NOTES

216 UNREADABLE' for $1.5 \mathrm{~s}$, depending on whether the neck position was assigned as the CS+, CS-,

217 or GS respectively. Each trial was followed by a $1 \mathrm{~s}$ interval before onset of the next trial. The

218 pain-expectancy judgments served as primary outcome for the evaluation of predictive learning 219 and stimulus generalization.

\section{**INSERT FIGURE 2**}

Figure 2. Flow chart of the predictive learning task, with a CS-, CS+, and GS trial as examples.

Middle row displays the question: To what extent do you expect Alex to experience pain in this position? CS $+=$ Conditioned Stimulus paired with "PAIN", CS- = Conditioned Stimulus paired with "NO PAIN", GS = Generalization Stimulus paired with "NOTES UNREADABLE". phase, a generalization phase, and an extinction phase (Table 2). The familiarization phase comprised one block, containing three trials. There was one $\mathrm{CS}+$ presentation, one CSpresentation, and one GS3 presentation. Stimuli were presented in randomized order. During the 
233 phase comprised four blocks, each containing eight trials. Each acquisition block included four

$234 \mathrm{CS}+$ presentations and four CS- presentations. Stimuli were presented in semi-randomized order, 235 ensuring that no more than two consecutive trials were of the same stimulus type. The CS+ was 236 followed by the 'PAIN' outcome in $75 \%$ of the trials, and by the 'NO PAIN' outcome in the other $23725 \%$ of the trials. The CS- was always followed by the 'NO PAIN' outcome. Which stimulus 238 served as the CS+ and CS- was counterbalanced between participants. The generalization phase 239 included two blocks, each containing seven trials. Each generalization block consisted of one CS+ 240 presentation, one CS- presentation and one presentation of each GS. Stimuli were presented 241 randomized within each block. The CS+ was again followed by the 'PAIN' outcome, whereas the 242 CS- was followed by the 'NO PAIN' outcome. The GS trials were followed by the outcome 243 'NOTES UNREADABLE' in order to prevent extinction. The extinction phase comprised five 244 blocks, each containing eight trials. Each extinction block included four CS+ presentations and 245 four CS- presentations. Stimuli were presented in semi-randomized order. During the extinction 246 phase, both the CS+ and the CS- were followed by the 'NO PAIN' outcome.

**INSERT TABLE $2 * *$

\section{Data analysis overview}

To test our first hypothesis as to whether people with persistent neck pain show less differential pain-expectancy judgments relative to pain-free controls, we conducted a $2 \times 2 \times 4$ Repeated Measures (RM) Analysis of Variance (ANOVA) with Group (patient/control) as between-subjects factor, and Stimulus type (CS+/CS-) and Block (ACQ1-4) as within-subjects factors. Planned between-group comparisons were then carried out to examine group differences in $\mathrm{CS}+$ and/or CS- responses. This analysis enabled us to first verify whether acquisition of 
256 differential conditioning occurred, this would be supported by a Stimulus Type x Block interaction.

257 Evidence supporting that the neck pain group showed less differential pain-expectancy learning,

258 would be provided by a significant interaction between Group and Stimulus Type (i.e. less

259 differential learning) and/or an interaction between Group, Stimulus Type, and Block (i.e. delayed

260 differential learning).

261

262

To test our second hypothesis that people with persistent neck pain would show overgeneralization relative to pain-free controls, we conducted a 2 × 2 × 7 RM ANOVA with

264 Group (patient/control) as between-subjects factor, and Stimulus type (CS+/GS1-5/CS-) and Block (GEN1-2) as within-subjects factors. Additionally, linear trend analyses were carried out to further investigate differences in stimulus generalization. To enable this, an index of the linear slope of generalisation was calculated for each participant CS+/GS1-5/CS- across the generalisation phase, using the Microsoft excel linear slope function. The slopes were then compared using an independent samples t-test. CS+ expectancy ratings were then compared to GS and CS- expectancy ratings within each group, to determine whether the CS expectancy ratings were distinct to other stimuli. Finally, these contrasts were repeated for the CS- expectancy ratings relative to the GS and CS expectancy ratings to further probe differential responding and generalization. HolmBonferroni corrections were applied to account for multiple comparisons (Abdi, 2010). Statistical support for overgeneralization in the neck pain group would firstly require observing a significant interaction between Group and Stimulus Type in the generalization phase. Secondly, observing a statistically flatter linear gradient of $\mathrm{CS}+/ \mathrm{GS} 1-5 / \mathrm{CS}$ - pain-expectancy judgments in the patient

277 group, representing higher ratings on the safe side of the gradient compared with the healthy

278 controls. Finally, follow-up comparisons specifically between CS- pain-expectancy and GS pain- 
279 expectancies within each group would further confirm non-differential responding in the patient

280 group. Specifically, we expect that more GS pain-expectancies will differ from CS- expectancies

281 in the control group relative to the patient group. The CS- vs. GS contrasts were emphasized

282 because the generalization decrement frequently renders CS+ expectancies different to all other

283 stimuli, making it less sensitive to detect between-group differences.

284

285

286

287

288

289

290

291

292

293

294

295

296

297

298

299

300

301

To test our third hypothesis as to whether people with persistent neck pain show impaired extinction as compared to pain-free controls, we conducted a 2 × 2 × 6 RM ANOVA with Group (patient/control) as between-subjects factor and Stimulus Type (CS+/CS-) and Block (ACQ4, EXT1-5) as within-subjects factors. Planned within-group comparisons comparing the CS+ to the CS- at the end of extinction as compared to the end of acquisition were carried out to confirm successful extinction within each group. To further evaluate group-based differences during extinction, we conducted planned between-group comparisons. The statistical criterion for delayed extinction was a significant Group x Stimulus Type x Block interaction, which would be indicative of a delayed elimination of the CS+/CS- differential expectancy ratings in the patient group.

For the conducted series of RM ANOVAs; Mauchly's test of sphericity was used to check whether the assumption of sphericity was violated. The Greenhouse-Geisser epsilon correction was reported together with corrected degrees of freedom and corrected $p$-values when this occurred. Partial eta squared was reported for RM ANOVA as a measure of effect size. Planned comparisons were corrected using the Holm-Bonferroni method (Holm, 1979) in case of multiple testing. Cohen's $d$ was reported as an index of effect for pairwise comparisons. Both JDW and DH conducted all statistical analyses, independently, with no significant conflict. One 
302 immaterial conflict arose due to differences in methods to calculate and compare the linear

303 slopes. Analyses were carried out with Statistica 13.0 (Statistica TIBCO. v. 13.3.0," 2017) and

304 JASP 0.9 (“JASP (version 0.9)[Computer Software],” 2018)

305

306

\section{Results}

Hypothesis 1: Do people with persistent neck pain show less differential acquisition in painexpectancy judgments than pain-free controls?

309 The analysis revealed a main effect of Stimulus Type, $F(1,58)=158.95, p<0.001, \eta_{p}^{2}=0.66$,

310 suggesting that for all participants pain-expectancy judgments for the CS+ were higher than for

311 the CS-. As expected, this difference significantly increased over time, Stimulus Type x Block

312 interaction, $F(3,174)=3.55, p<0.05, \eta_{p}^{2}=0.06, \varepsilon=0.84$ (see Figure 3 ). Differential pain-

313 expectancy judgments were significantly smaller in the patient group, $\Delta(\mathrm{CS}+, \mathrm{CS}-)$ controls $=6.05$

$314(S D=2.67)$ and patients $=2.68(S D=2.69)$, Stimulus Type x Group interaction, $F(1,58)=23.74$,

$315 p<0.001, \eta_{p}^{2}=0.10$. This effect was not modulated by time, Stimulus Type x Block x Group

316 interaction, $F(3,174)=0.13, p=0.92, \eta_{p}^{2}=0.00$

317 Analysis across acquisition revealed that patients displayed significantly lower CS+ painexpectancies, $t(58)=3.86, p<0.001$, Cohen's $d=1.00, M_{\text {patients }}(S D)=5.11(2.30) v s . M_{\text {controls }}$

$319(\mathrm{SD})=7.18(1.83)$, and significantly higher CS- pain-expectancies, $t(58)=2.48, p=0.016$, 320 Cohen's $d=0.64, M_{\text {patients }}(S D)=2.43(2.53) v s . M_{\text {controls }}(\mathrm{SD})=1.13(1.35)$.

321 As expected, these findings show that people with persistent neck pain show less differential pain322 expectancy judgments due to heightened pain-expectancy judgments for safety cues. In addition, 
323 and unexpectedly, lower pain-expectancy judgments for danger cues also contribute to the reduced

324 differential predictive learning.

**INSERT FIGURE 3**

Figure 3. Mean pain-expectancy judgments for the CS+ and CS- both for the pain-free control group $(n=30)$ and persistent neck pain group $(n=30)$ during the familiarization phase (FAM), the four blocks of acquisition (ACQ1-4), and the five blocks of extinction (EXT1-5), separately per block. Vertical bars represent $95 \%$ confidence intervals. CS $+=$ Conditioned Stimulus paired with "PAIN" in 75\% of the trials, CS- = Conditioned Stimulus always paired with "NO PAIN".

judgments compared to pain-free controls?

We tested the generalization of pain-expectancy to novel but similar GSs and found a significant

337 main effect of Stimulus Type, $F(2.82,163.32)=50.22, p<0.001, \eta_{p}^{2}=0.46$, and a significant

338 interaction effect of Stimulus Type x Group, $F(2.82,163)=7.53, p<0.001, \eta_{p}^{2}=0.12$, suggesting

339 group differences in pain-expectancy judgments (see Figure 4). To probe group differences across

340 the generalization phase, we first compared generalization gradients among groups, using linear

341 trends generated by including CS+, GS1-5, and CS- expectancy judgments. The analyses revealed

342 that patients display flatter generalization gradients compared with controls, $t(58)=4.07, p<$

0.001, Cohen's $d=1.05, M_{\text {patients }}(S D)=-0.31(0.72) v s . M_{\text {controls }}(\mathrm{SD})=-1.12(0.82)$. 
345 Finally, CS+ and CS- expectancy ratings were compared to all other expectancy ratings within 346 each group. Holm-Bonferroni corrected comparisons showed that CS+ expectancy ratings were 347 significantly greater than all other stimuli within both groups (all $p s<0.015$ ), showing that both 348 groups had significantly high expectancy during the CS+ relative to all other stimuli, this is 349 generally referred to as a generalization decrement. For the contrasts relative to the CS-, only the $\mathrm{CS}+$ expectancy was different $(p=0.006)$ in the patient group (all other $p s>0.12)$. In the controls,

351 however, the GS1 and GS2 expectancy ratings were also significantly different to the CS- (ps < 352 0.001), suggesting that pain-expectancy ratings elicited by these stimuli were higher than for the 353 safe neck position (CS-). This finding further reflects non-differential responding in the patient 354 group.

\section{**INSERT FIGURE 4**}

Figure 4. Mean pain-expectancy judgments for CS+, GS1-5, CS- for the patient group (n=30) as well as the pain-free controls $(n=30)$ during the generalization phase, averaged over blocks. Vertical bars represent 95\% confidence intervals. CS $+=$ Conditioned Stimulus paired with "PAIN" in $75 \%$ of the trials, CS- = Conditioned Stimulus always paired with "NO PAIN", GS1 = Generalization Stimulus most closely resembling the CS+. 
365 The analysis of the pain-expectancy judgments during extinction yielded a main effect of

366 Stimulus Type, $F(1,58)=37.09, p<0.001, \eta_{p}^{2}=0.34$, and a significant Stimulus Type $\mathrm{x}$ Block

367 interaction, $F(4,232)=30.25, p<0.001, \eta_{p}^{2}=0.32$, likely reflecting diminishing CS +

368 expectancies, as compared to the CS-, across extinction blocks relative to the end of acquisition.

369 In addition, we observed a significant Stimulus Type x Group interaction, $F(1,58)=13.69, p<$

$3700.001, \eta_{p}^{2}=0.13$, and a significant Stimulus Type x Block x Group interaction, $F(4,232)=6.09$,

$371 p=0.01, \eta_{p}^{2}=0.06$. Visual inspection of the data (Figure 3) indicated that the interaction might

372 be driven by seemingly quicker extinction of (the already lesser) differential expectancies in the

373 patient group. While no group differences during Extinction block 1, a Stimulus Type x Group

374 RM ANOVA within Extinction block 2 was consistent with group differences $F(1,58)=2.29, p$

$375<0.001, \eta_{p}^{2}=0.26$. Inspection of Figure 3 suggests that this effect was due to extinction of

376 differential expectancies in the patient group. In the final extinction block, neither groups

377 retained differential (CS+ vs. CS-) pain-expectancies, Controls: $t(29)=1.65, p=0.11$; Patients:

$378 t(29)=0.39, p=0.70$, with no group differences in CS + ratings, $t(58)=-1.03, p=0.31)$ or CS-

379 ratings, $t(58)=-1.76, p=0.08$.

\section{Discussion}

382 In this study we investigated whether people with persistent neck pain show deficits in predictive

383 learning relative to pain-free controls. We hypothesized that people with persistent neck pain 384 would show (1) less differential pain-expectancy learning, (2) overgeneralization of pain385 expectancies to novel but similar neck postures, and (3) reduced extinction of differential pain386 expectancies. In line with our first hypothesis, we observed less differential pain-expectancies for 
387 the CS+ and the CS- for the patients as compared to controls. These results are broadly consistent

388 with earlier findings on patients with chronic unilateral hand pain (Meulders et al., 2014),

389 fibromyalgia (Meulders et al., 2015; Meulders et al., 2017) and chronic back pain (Klinger et al.,

390 2010; Schneider et al., 2004). This finding appeared to be both due to reduced CS+ expectancy,

391 and greater CS- expectancy in the neck pain group. With regards to our second hypothesis that

392 people with neck pain would overgeneralize pain-expectancies relative to pain-free controls,

393 results were partially aligned with our hypothesis. Here, both groups showed greater CS+

394 expectancy relative to all other stimuli, reflecting a common generalization decrement. However,

395 when considering CS- expectancy, patients did not appear to discriminate it from any of the GSs,

396 whereas controls did. This reduced discrimination among cues in the generalization phase was also

397 consistent with the flatter generalization gradient in the patient group, providing supporting

398 evidence for group differences in generalization. The possibility that people with neck pain tend

399 to overgeneralize pain-expectancy is in line with existing literature on pain-expectancy judgments

400 in people with persistent pain, including fibromyalgia and chronic hand pain patients (Meulders et

401 al., 2014; Meulders et al., 2017). It is also interesting to note that in general responses to both the

402 CSs and the GSs were lower for patients than in the healthy control group. Such blunted fear

403 responding and wider fear generalization gradients have been recently observed in people who

404 experienced childhood maltreatment (Lonsdorf, 2019). Because differences in differential learning

405 can influence generalization, we explored whether acquisition phase differential learning mediated

406 generalization linear slopes (see Supplementary file for full details). Direct and indirect (mediated)

407 effects of group were of approximately equal weight, suggesting that as least part of the

408 generalization effects was independent of acquisition learning. Contrary to our third hypothesis-

409 that people with neck pain would show delayed extinction of differential learning — differential 
410 responding appeared to disappear quicker in people with neck pain. The rate of extinction learning

411 however is likely not different from healthy controls, patients simply seem to extinguish quicker

412 because they start off with less differential responding and overall lower responses at the end of

413 acquisition. It is notable that although delayed extinction is commonly identified as a possible

414 impairment contributing to the maintenance of chronic pain, there is currently little supporting

415 evidence for the thesis (Harvie et al., 2017; also see Schneider et al., 2004).

\section{Differential learning}

418 The most robust finding in this study was the reduced differential learning in people with persistent neck pain relative to pain-free controls. This reduced differential learning was driven by both lower pain-expectancies for the CS+, and higher pain-expectancy for the CS-. In prior studies, in people with persistent pain and people with anxiety disorders (Harvie et al., 2017; Lissek et al., 2005), reduced differential learning is typically the primary result of heightened CS- expectancies-

423 referred to as impaired safety learning. The current study shows preliminary evidence that safety learning deficits are present in some individuals with neck pain. The deficits in differential learning 425 indicate a tendency towards reduced efficiency in selectively evaluating safety cues. That is, 426 patients in general may be able to learn when to expect pain, and when not too, but with greater 427 uncertainty. With regards to the lower CS+ pain-expectancy judgements, a similar data pattern 428 (reduced responding to CS+ in patients compared to healthy controls, albeit not significant) was observed previously (Meulders et al., 2014), we speculate that expectancy judgements may be 430 contaminated by certainty judgements. That is, one may have a high degree of pain-expectancy, 431 but a low degree of certainty. For example, one might expect rain, but lack certainty regarding that 432 judgment. This example shows that expectancy and certainty do not necessarily converge. This 
433 lack of certainty would result in people tempering their CS+ expectancy ratings relative to if they

434 had both a high degree of expectancy and a high degree of certainty. Greater overall uncertainty

435 in patients may arise from factors such as reduced self-efficacy/self-confidence in addition to

436 impaired predictive learning.

\section{Limitations and future directions}

439 The results of this study are subject to several limitations. The cross-sectional nature of the design

440 limits our capacity to reveal causal relationships between the chronic pain status and altered

441 expectancy learning outcomes. It is thereby unclear whether these deficits contribute to chronic

442 pain, or are a result of it. Indeed, prospective studies are required in this endeavor. Notably, the

443 greatest deficits may occur in the most severely afflicted patients. The patients in the current study

444 in general had only mild-moderate severity with respect to pain and disability. This may in part be

445 due to the exclusion of subjects on opioid medications. While this may have excluded more severe

446 patients, it strengthened the study in preventing the confounding effect of opioids on cognitive

447 performance. Further, participants showed no, or low levels, of co-morbid psychological

448 dysfunction, which may be critical drivers of the constructs under investigation. Crucially, this is

449 different to other studies where participants did show significant group differences in

450 psychological variables (e.g. Meulders et al., 2014; Meulders et al., 2015; Meulders et al., 2017).

451 That we nonetheless found group differences in predictive learning, despite less disability and

452 psychological dysfunction compared to previously studied patient groups, is remarkable and raises

453 the possibility that learning deficits may exist independent of psychological factors and may be

454 present even in less severe cases of persistent pain. Finally, as we have disproportionately 
455 pioneered this line of research, replication from independent labs is needed, and indeed beginning

456 to emerge (Both, Brauer, Weijenborg, \& Laan, 2017; Heathcote et al., 2020).

457

458

459 Conclusions

460 We aimed to determine whether people with chronic neck pain show deficits in expectancy

461 learning relative to pain-free controls. We found evidence for our first hypothesis observing less

462 differential pain-expectancies for the people with persistent neck pain as compared to pain-free

463 controls, suggesting reduced efficacy in identifying predictors of pain. Some preliminary support

464 was also found for overgeneralization of pain-expectancies relative to controls, in that

465 generalization gradients were flatter in the patient group. Contrary to our final hypotheses, people

466 with neck pain did not show reduced extinction learning. These findings may be relevant to

467 understanding behavioral aspects of chronic pain, however prospective studies are needed.

468

469 
470

471

472

473

474

475

476

477

478

479

480

481

482

483

484

485

486

487

488

489

490

\section{References}

Abdi, H. (2010). Holm's sequential Bonferroni procedure. Encyclopedia of Research Design,

$$
1(8), 1-8 .
$$

Apergis-Schoute, A., Gillian, C. M., Fineberg, N. A., Fernandez-Egea, E., Sahakian, B. J., \& Robbins, T. W. (2017). Neural basis of impaired safety signaling in obsessive compulsive disorder. Proceedings of the National Academy of Sciences, 114, 3216-3221. https://doi.org/10.1073/pnas.1609194114

Boddez, Y., Baeyens, F., Luyten, L., Vansteenwegen, D., Hermans, D., \& Beckers, T. (2013). Rating data are underrated: Validity of US expectancy in human fear conditioning. Journal of Behavior Therapy and Experimental Psychiatry, 44(2), 201-206. https://doi.org/10.1016/j.jbtep.2012.08.003

Clifton, N. E., Pocklington, A. J., Scholz, B., Rees, E., Walters, J. T. R., Kirov, G., ... Hall, J. (2017). Schizophrenia copy number variants and associative learning. Molecular Psychiatry, 22(2), 178-182. https://doi.org/10.1038/mp.2016.227

Crombez, G., Vlaeyen, J. W. S., Heuts, P. H. T. G., \& Lysens, R. (1999). Pain-related fear is more disabling than pain itself: Evidence on the role of pain-related fear in chronic back pain disability. Pain, 80(1-2), 329-339. https://doi.org/10.1016/S0304-3959(98)00229-2

Dickinson, A. (1980). Contemporary animal learning theory. Cambridge: Cambridge University Press.

Duits, P., Cath, D. C., Lissek, S., Hox, J. J., Hamm, A. O., Engelhard, I. M., ... Baas, J. M. P. (2015). Updated meta-analysis of classical fear conditioning in the anxiety disorders. 
492

493

494

495

496

497

498

499

500

501

502

503

504

505

506

507

508

509

510

511

Dymond, S., Dunsmoor, J. E., Vervliet, B., Roche, B., \& Hermans, D. (2015). Fear

Generalization in humans: systematic review and implications for anxiety disorder research. Behavior Therapy, 46(5), 561-582. https://doi.org/10.1016/j.beth.2014.10.001

Enquist, M., Lind, J., \& Ghirlanda, S. (2016). The power of associative learning and the ontogeny of optimal behaviour. Royal Society Open Science, 3(11). https://doi.org/10.1098/rsos.160734

Grillon, C. (2002). Associative learning deficits increase symptoms of anxiety in humans. Biological Psychiatry, 51, 851-858.

Harvie, D., Moseley, G., Hillier, S., \& Meulders, A. (2017). Classical conditioning differences associated with chronic pain: a systematic review. Journal of Pain, 18(8), 889-898. https://doi.org/10.1016/j.jpain.2017.02.430

Holm, S. (1979). A simple sequentially rejective multiple test procedure. Scandinavian Journal of Statistics, 6(2), 65-70.

Honig, W. K., \& Urcuioli, P. J. (1981). The legacy of Guttman and Kalish (1956): 25 years of research on stimulus generalization. Journal of the Experimental Analysis of Behavior, 36(3), 405-445. https://doi.org/10.1901/jeab.1981.36-405

Houwer, J. De, \& Beckers, T. (2002). A review of recent developments in research and theories on human contingency learning. The Quarterly Journal Of Experimental Psychology, 55(4), 289-310. https://doi.org/10.1080/0272499024400003

JASP (version 0.9)[Computer Software]. (2018). Retrieved from https://jasp-stats.org/ 
512 Jenewein, J., Moergeli, H., Sprott, H., Honegger, D., Brunner, L., Ettlin, D., ... Hasler, G.

513 (2013). Fear-learning deficits in subjects with fibromyalgia syndrome? European Journal of

514 Pain, 17(9), 1374-1384. https://doi.org/10.1002/j.1532-2149.2013.00300.x

515 Jovanovic, T., Kazama, A., Bachevalier, J., \& Davis, M. (2012). Impaired safety signal learning

516 may be a biomarker of PTSD. Neuropharmacology, 62(2), 695-704.

$517 \quad$ https://doi.org/10.1016/j.neuropharm.2011.02.023

518 Kalish, H. (1969). Stimulus generalization. In M. H. M. (Ed.), Learning: Processes (pp. 207519 297). Oxford, England: Macmillan.

520 Klinger, R., Matter, N., Kothe, R., Dahme, B., Hofmann, U. G., \& Krug, F. (2010).

521 Unconditioned and conditioned muscular responses in patients with chronic back pain and

522 chronic tension-type headaches and in healthy controls. Pain, 150(1), 66-74.

$523 \quad$ https://doi.org/10.1016/j.pain.2010.03.036

524 Kroenke, K., Spitzer, R. L., \& Williams, J. B. W. (2001). The PHQ-9. Journal of General 525 Internal Medicine, 16(9), 606-613. https://doi.org/10.1046/j.1525-1497.2001.016009606.x

526 Landers, M. R., Creger, R. V., Baker, C. V., \& Stutelberg, K. S. (2008). The use of fear-

527 avoidance beliefs and nonorganic signs in predicting prolonged disability in patients with

528 neck pain. Manual Therapy, 13(3), 239-248. https://doi.org/10.1016/j.math.2007.01.010

529 Lissek, S., Powers, A. S., McClure, E. B., Phelps, E. A., Woldehawariat, G., Grillon, C., \& Pine,

530 D. S. (2005). Classical fear conditioning in the anxiety disorders: A meta-analysis.

531 Behaviour Research and Therapy, 43(11), 1391-1424.

$532 \quad$ https://doi.org/10.1016/j.brat.2004.10.007 
533 Both, S., Brauer, M., Weijenborg, P., \& Laan, E. (2017). Effects of aversive classical

534 conditioning on sexual response in women with dyspareunia and sexually functional

535 controls. The journal of sexual medicine, 14(5), 687-701.

536 Heathcote, L. C., Timmers, I., Kronman, C. A., Mahmud, F., Hernandez, J. M., Bentley, J., . . .

537 Simons, L. E. (2020). Brain signatures of threat-safety discrimination in adolescent

$538 \quad$ chronic pain. Pain, 161(3), 630-640.

539 Lonsdorf, T. B. (2019, May 2019). More than just noise: Individual differences in fear

540 acquisition, extinction and the return of fear and methodological considerations derived

541 from a meta-research perspective. Paper presented at the APS Annual Convention,

$542 \quad$ Washington, D.C.

543 Meulders, A., Harvie, D. S., Bowering, J. K., Caragianis, S., Vlaeyen, J. W., \& Moseley, G. L.

544 (2014). Contingency learning deficits and generalization in chronic unilateral hand pain

545 patients. The Journal of Pain, 15(10), 1046-1056.

546 Meulders, A., Jans, A., \& Vlaeyen, J. W. (2015). Differences in pain-related fear acquisition and

547 generalization: an experimental study comparing patients with fibromyalgia and healthy

$548 \quad$ controls. Pain, 156(1), 108-122.

549 Meulders, A. (2019). From fear of movement-related pain and avoidance to chronic pain

550 disability: a state-of-the-art review. Current Opinion in Behavioral Sciences, 26, 130-136.

$551 \quad$ https://doi.org/10.1016/j.cobeha.2018.12.007

552 Meulders, A., Harvie, D. S., Bowering, J. K., Caragianis, S., Vlaeyen, J. W. S., \& Moseley, G. L.

553 (2014). Contingency learning deficits and generalization in chronic unilateral hand pain

554 patients. The Journal of Pain, 15(10), 1046-1056.

$555 \quad$ https://doi.org/10.1016/j.jpain.2014.07.005 
556 Meulders, A., Jans, A., \& Vlaeyen, J. W. S. (2015). Differences in pain-related fear acquisition

557 and generalization: an experimental study comparing patients with fibromyalgia and healthy

558 controls. Pain, 156, 108-122. https://doi.org/10.1016/j.pain.0000000000000016

559 Meulders, A., Meulders, M., Stouten, I., De Bie, J., \& Vlaeyen, J. W. S. (2017). Extinction of

560 fear generalization: a comparison between fibromyalgia patients and healthy control

561 participants. Journal of Pain, 18(1), 79-95. https://doi.org/10.1016/j.jpain.2016.10.004

562 Nederhand, M. J., Ijzerman, M. J., Hermens, H. J., Turk, D. C., \& Zilvold, G. (2004). Predictive

563 value of fear avoidance in developing chronic neck pain disability: Consequences for

$564 \quad$ clinical decision making. Archives of Physical Medicine and Rehabilitation, 85(3), 496-

565 501. https://doi.org/10.1016/j.apmr.2003.06.019

566 Pavlov, I. P. (1927). Conditioned reflexes. London: Oxford University Press.

567 Schneider, C., Palomba, D., \& Flor, H. (2004). Pavlovian conditioning of muscular responses in

568 chronic pain patients: central and peripheral correlates. Pain, 112(3), 239-247.

569 https://doi.org/10.1016/j.pain.2004.08.025

570 Shanks, D. R., \& Dickinson, A. (1988). Associative Accounts of Causality Judgment.

$571 \quad$ Psychology of Learning and Motivation, 21, 229-261. https://doi.org/10.1016/S0079-

$572 \quad 7421(08) 60030-4$

573 Spielberger, C. D., Gorsuch, R. L., Lushene, R., Vagg, P. R., \& Jacobs, G. A. (1983). Manual for

574 the State-Trait Anxiety Inventory. Palo Alto, CA: Consulting Psychologists Press.

575 Spruyt, A., Clarysse, J., Vansteenwegen, D., Baeyens, F., \& Hermans, D. (2010). Affect 4.0.

576 Experimental Psychology, 57(1), 36-45. https://doi.org/10.1027/1618-3169/a000005 
577 Statistica TIBCO. v. 13.3.0. (2017). TIBCO Software Inc, Palo Alto, CA, USA. Retrieved from

578 https://www.tibco.com/products/tibco-statistica

579 Sullivan, M., Bishop, S., \& Pivik, J. (1995). The pain catastrophizing scale: user manual.

$580 \quad$ Psychological Assessment, 7(4), 524-532. https://doi.org/10.1037/1040-3590.7.4.524

581 Vernon, H., \& Mior, S. (1991). The Neck Disability Index: a study of reliability and validity.

582 Journal of Manipulative and Physiological Therapeutics, 14(7), 409-415.

583 Vlaeyen, J. W. S. (2015). Learning to predict and control harmful events. Pain, 156, S86-S93.

584 https://doi.org/10.1097/j.pain.0000000000000107

585 Vlaeyen, J. W. S., \& Linton, S. J. (2012). Fear-avoidance model of chronic musculoskeletal pain:

58612 years on. Pain, 153(6), 1144-1147. https://doi.org/10.1016/j.pain.2011.12.009

587 Vos, T., Barber, R. M., Bell, B., Bertozzi-Villa, A., Biryukov, S., Bolliger, I., ... Murray, C. J. L.

588 (2015). Global, regional, and national incidence, prevalence, and years lived with disability

589 for 301 acute and chronic diseases and injuries in 188 countries, 1990-2013: a systematic

$590 \quad$ analysis for the Global Burden of Disease Study 2013. The Lancet, 386(9995), 743-800.

$591 \quad$ https://doi.org/10.1016/S0140-6736(15)60692-4

592 Watson, D., Clark, L. A., \& Tellegen, A. (1988). Development and validation of brief measures

593 of positive and negative Affect: the PANAS scales. Journal of Personality and Social

594 Psychology, 54(6), 1063-1070. https://doi.org/10.1051/epjconf/201714006017

595 Woby, S. R., Roach, N. K., Urmston, M., \& Watson, P. J. (2005). Psychometric properties of the

596 TSK-11: a shortened version of the Tampa Scale for Kinesiophobia. Pain, 117(1-2), 137-

597 144. https://doi.org/10.1016/j.pain.2005.05.029 
598

599

600

601

602

603

Peer) reviewing PDF | (2019:12:43686:1:1:CHECK 8 May 2020) 


\section{Table $\mathbf{1}$ (on next page)}

Demographics and participant characteristics for both the persistent neck pain patients and pain-free controls 
Table 1. Demographics and participant characteristics for both the persistent neck pain patients and pain-free controls

\begin{tabular}{llllllll}
\hline & \multicolumn{3}{l}{ Persistent neck pain $(n=30)$} & \multicolumn{3}{l}{ Pain-free controls $(n=30)$} & Mean diff. $(95 \% \mathrm{CI})$ \\
\cline { 2 - 9 } & Mean & $S D$ & Range & Mean & $S D$ & Range & \\
\hline Age (in years) & 50.90 & 15.87 & $18-72$ & 49.90 & 14.55 & $23-73$ & $-1.00[-0.34,-1.66]$ \\
PANAS - Positive affect & 31.60 & 8.06 & $19-48$ & 30.73 & 7.68 & $14-47$ & $-0.87[1.94,-3.67]$ \\
PANAS - Negative affect & 12.50 & 3.18 & $10-21$ & 12.53 & 3.57 & $10-23$ & $0.03[1.26,-1.20]$ \\
STAI-T - Total & 38.87 & 10.65 & $21-58$ & 36.60 & 10.89 & $20-63$ & $-2.27[1.68,-6.22]$ \\
PCS - Total & 13.93 & 10.04 & $2-36$ & 7.97 & 7.03 & $0-32$ & $-5.97[-2.81,-9.12]$ \\
PCS - Rumination & 4.3 & 3.54 & $0-14$ & 3.17 & 3.13 & $0-12$ & $-1.13[0.12,-2.39]$ \\
PCS -Magnification* & 3.07 & 3.04 & $0-10$ & 1.63 & 1.92 & $0-8$ & $-1.43[-0.48,-2.38]$ \\
PCS -Helplessness** & 6.57 & 4.15 & $1-15$ & 3.17 & 2.74 & $0-12$ & $-3.40[-2.16,-4.64]$ \\
PHQ-9* - Total & 7.47 & 5.64 & $0-18$ & 3.9 & 4.49 & $0-20$ & $-5.97[-2.81,-9.12]$ \\
TSK-11 - Total** & 24.10 & 5.49 & $12-36$ & 18.97 & 5.18 & $11-28$ & $-5.13[-3.24,-7.03]$ \\
TSK-11 - Somatic Focus & 10.77 & 3.03 & $5-17$ & 8.63 & 2.86 & $5-14$ & $-2.13[-1.04,-3.23]$ \\
TSK-11 - Avoidance & 13.33 & 3.25 & $7-23$ & 10.33 & 2.60 & $6-15$ & $-3.00[-1.98,-4.02]$ \\
NDI - Total & $30.0 \%$ & 12.4 & $12-54 \%$ & -- & -- & -- & \\
Current pain (0-10) & 4.07 & 2.21 & $0-8$ & 0.1 & 0.31 & $0-1$ & $-3.97[-3.38,-4.55]$ \\
Av. pain last 2 weeks (0-10) & 4.47 & 1.18 & $0-7$ & 0.13 & 0.43 & $0-2$ & $-4.33[-3.84,-4.83]$ \\
Worst pain 2 weeks (0-10) & 6.8 & 2.53 & $0-10$ & 0.57 & 1.14 & $0-7$ & $-6.27[-5.52,-7.01]$ \\
\hline
\end{tabular}

${ }^{*} p<.05, * * p<.01 * *, P A N A S=$ Positive and Negative Affect Schedule, STAI-T = Trait version of the State-Trait Anxiety Inventory, PCS = pain pain-catastrophizing scale, PHQ-9 patient health questionnaire, TSK-11 = Tampa scale for kinesiophobia, NDI = Neck Disability Index, Range = the minimum and maximum value for the given variable 
Table 2 (on next page)

Experimental design 
1 Table 2. Experimental design

\begin{tabular}{lrrr}
\hline Familiarization & Acquisition* & Generalization & Extinction \\
\hline$\left(\begin{array}{l}1 \times \mathrm{CS}+ \\
1 \times \mathrm{x} 3 \\
1 \times \mathrm{CS}-\end{array}\right)$ & $4 \times\left(\begin{array}{l}4 \times \mathrm{CS}+ \\
4 \times \mathrm{CS}-\end{array}\right)$ & $2 \times\left(\begin{array}{l}1 \times \mathrm{CS}+ \\
1 \times \mathrm{xS} 1-5 \\
1 \times \mathrm{CS}-\end{array}\right)$ & $5 \times\left(\begin{array}{l}4 \times \mathrm{CS}+ \\
4 \times \mathrm{CS}-\end{array}\right)$
\end{tabular}




\section{Figure 1}

Figure 1

Conditioned stimuli (CS+ and CS-) and generalization stimuli (GS1-5) presented with CSs on the extremes and the intermediate GSs in between representing the generalization gradient. Which stimulus served as the CS+ and the CS- (i.e. white vs. blue labels) was counterbalanced across participants.

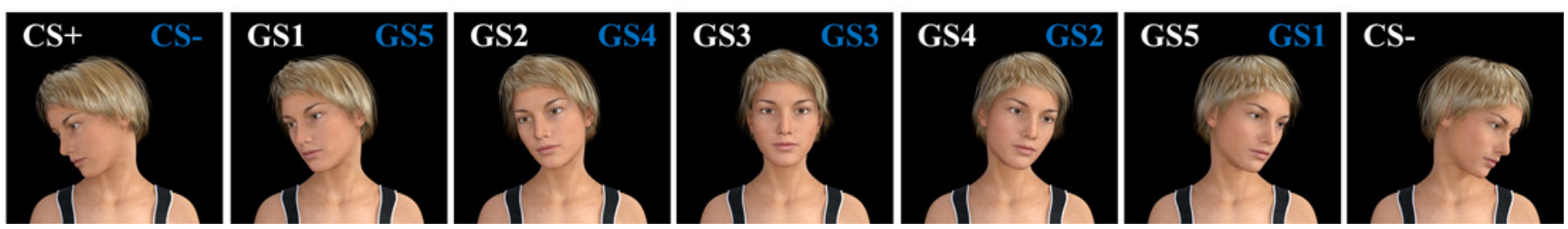




\section{Figure 2}

Figure 2

Flow chart of the predictive learning task, with a CS-, CS+, and GS trial as examples. Middle row displays the question: To what extent do you expect Alex to experience pain in this position? CS $+=$ Conditioned Stimulus paired with "PAIN", CS- = Conditioned Stimulus paired with "NO PAIN", GS = Generalization Stimulus paired with "NOTES UNREADABLE".

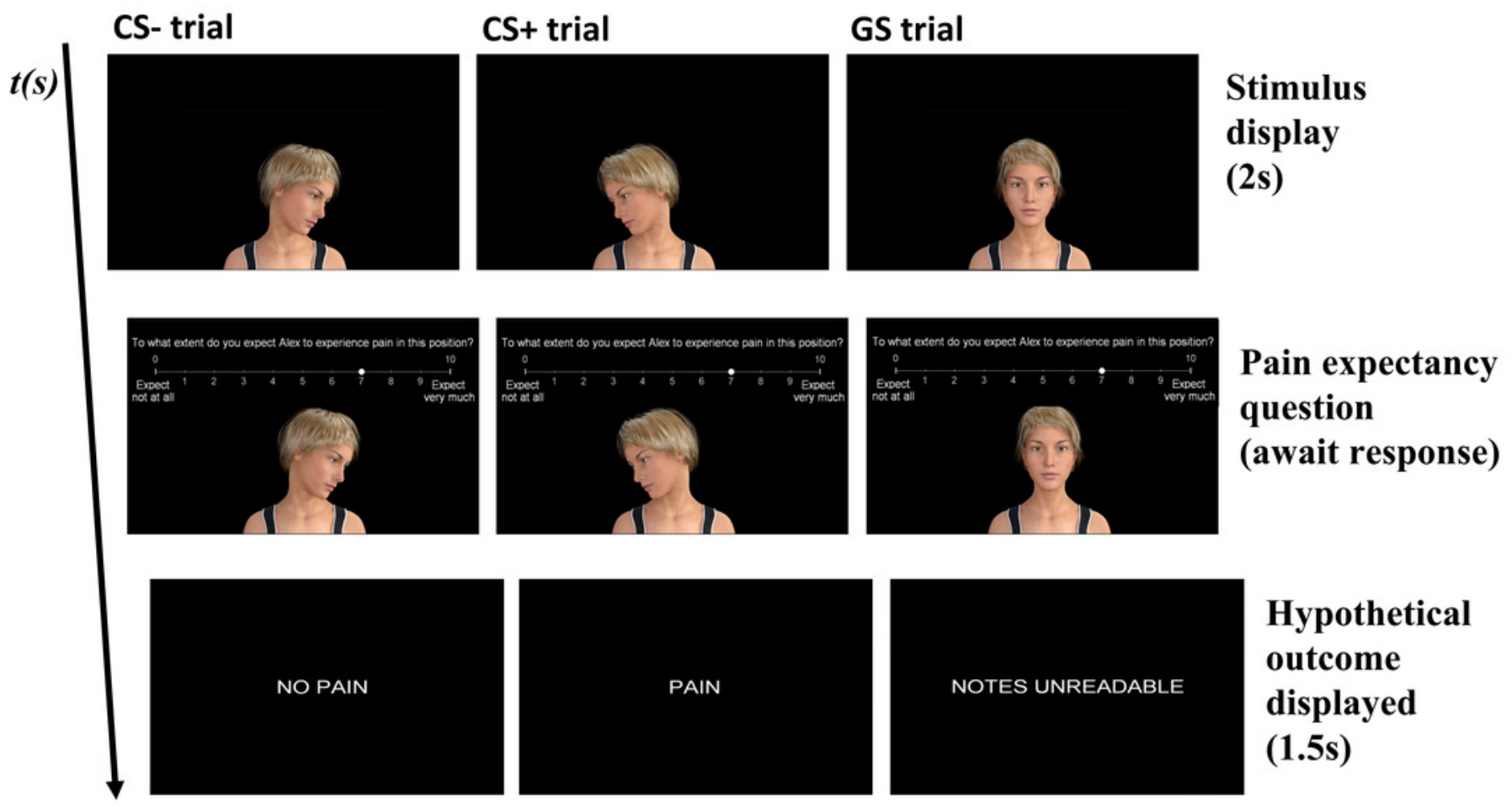




\section{Figure 3}

Figure 3

Mean pain-expectancy judgments for the CS+ and CS- both for the pain-free control group $(n=30)$ and persistent neck pain group $(n=30)$ during the familiarization phase (FAM), the four blocks of acquisition (ACQ1-4), and the five blocks of extinction (EXT1-5), separately per block. Vertical bars represent $95 \%$ confidence intervals. CS $+=$ Conditioned Stimulus paired with "PAIN" in 75\% of the trials, CS- = Conditioned Stimulus always paired with "NO PAIN". 


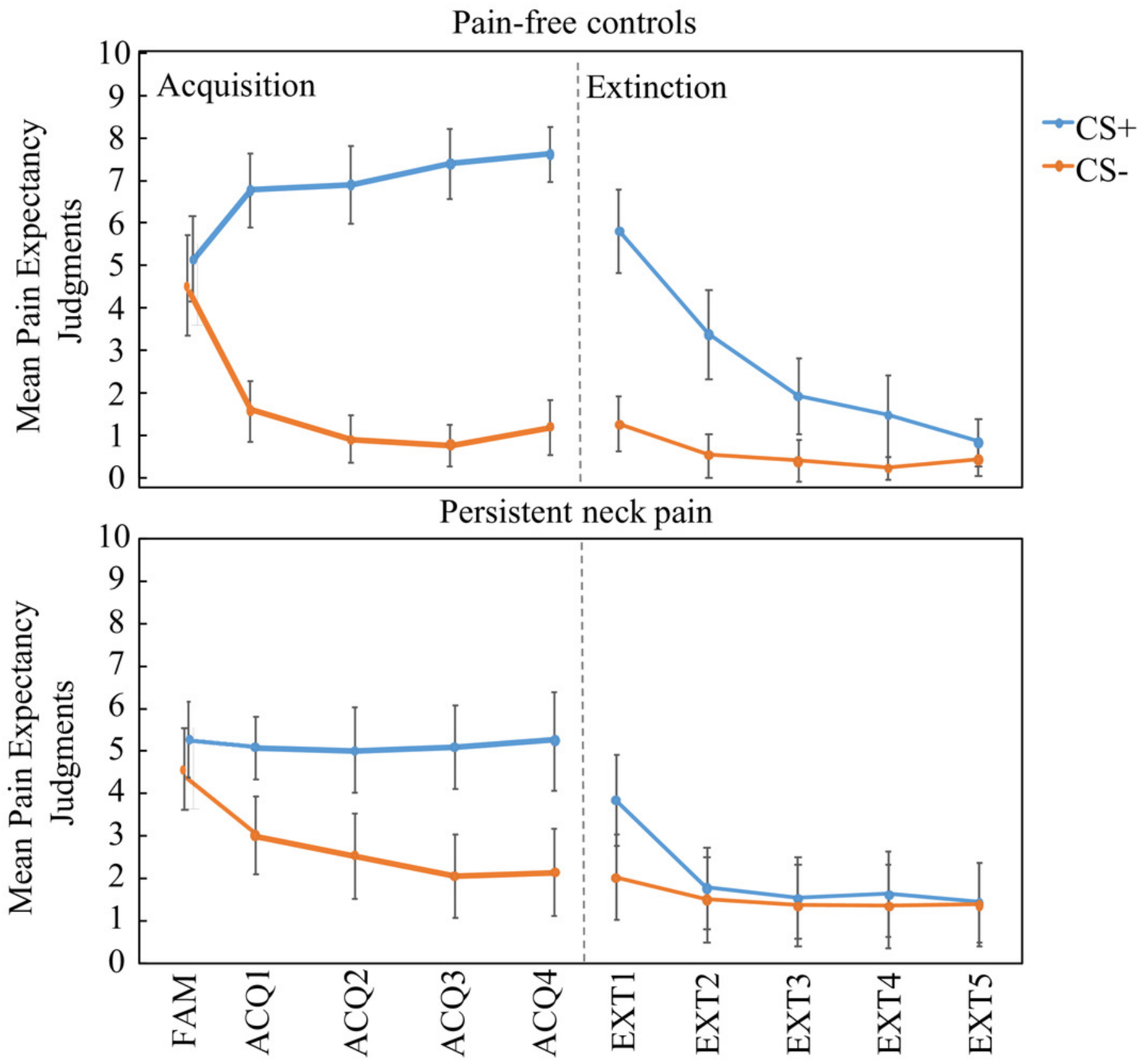


Figure 4

Figure 4

Mean pain-expectancy judgments for $\mathrm{CS}+, \mathrm{GS1}-5, \mathrm{CS}$ - for the patient group $(\mathrm{n}=30)$ as well as the pain-free controls $(n=30)$ during the generalization phase, averaged over blocks. Vertical bars represent $95 \%$ confidence intervals. CS+ $=$ Conditioned Stimulus paired with "PAIN" in $75 \%$ of the trials, CS- $=$ Conditioned Stimulus always paired with "NO PAIN", GS1 = Generalization Stimulus most closely resembling the CS+.

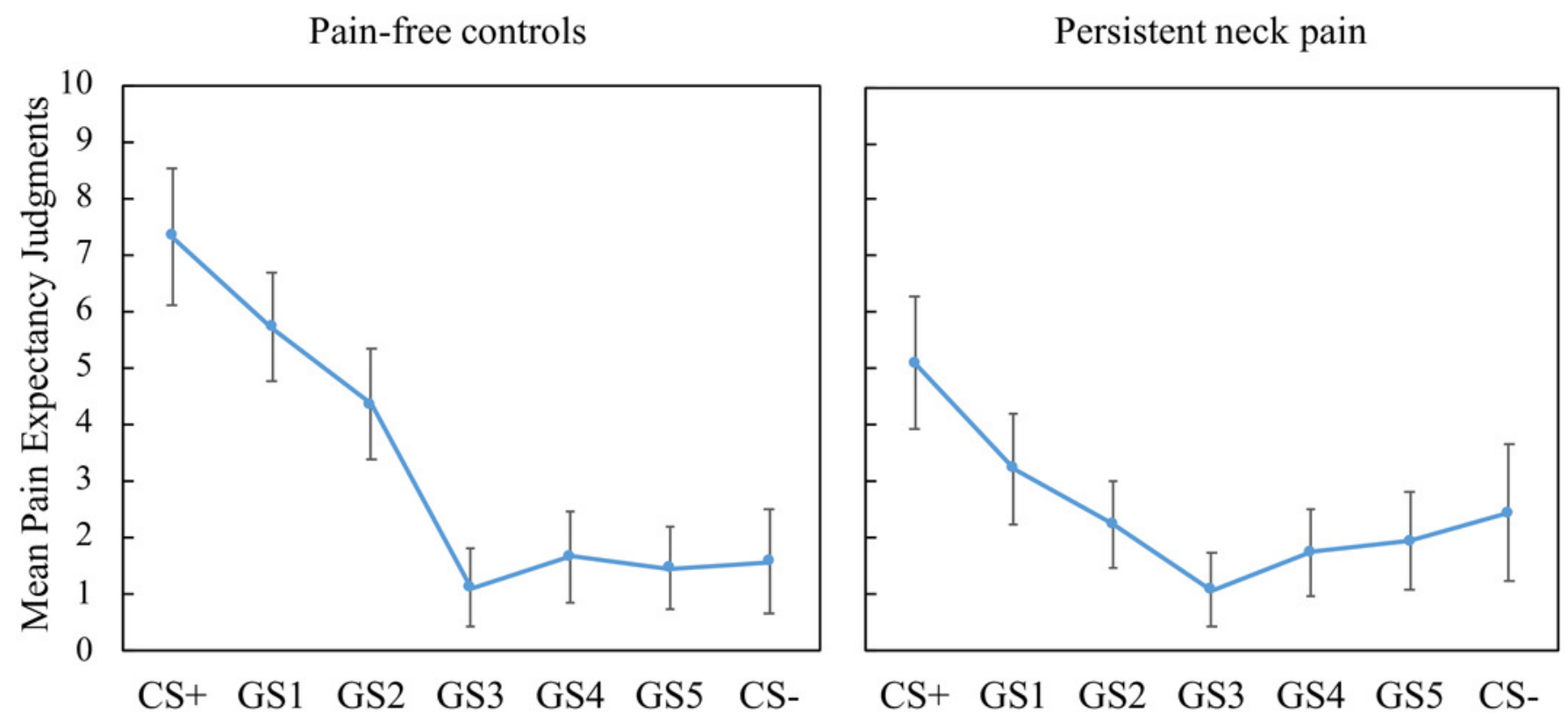

